\title{
Clinical development of RET inhibitors in RET-rearranged non-small cell lung cancer: Update
}

\author{
Luis Mendoza \\ IQVIA, Prague, Czech Republic
}

\begin{abstract}
Precision oncology is now the evidence-based standard of care for the management of many advanced non-small cell lung cancers (NSCLC). Notably, new molecular profiling technologies have permitted dynamic growth in the identification of actionable driver oncogenes including RET rearrangements. RET oncogenes cannot be adequately detected by immunohistochemistry, although fluorescence in situ hybridization, reverse transcriptase polymerase chain reaction and next-generation sequencing are complementary diagnostic tools. In the clinical setting, the benefit of the most developed RET inhibitors, i.e., cabozantinb, vandetanib and lenvatinb, in terms of response and median progressionfree survival has been demonstrated. The absence of striking clinical results of RET inhibitors underscores the clear need for development of more selective and potent RET inhibitors. This paper reviews the clinical data available on RET inhibitors in RET-associated NSCLC.
\end{abstract}

\section{Introduction}

In NSCLC, the main potentially targetable chromosome rearrangements involve the ALK, ROS1, NTRK and RET (rearranged during transfection) genes. However, these chromosomal rearrangements are present only in a small percentage of patients with lung cancer $\left(3 \%-7 \%, 1 \%-2 \%,{ }^{2} 3.3 \%{ }^{3}\right.$ and $\sim 1 \%$ $2 \%,{ }^{4}$ respectively). Oncogenic gene rearrangements in NSCLC can lead to the expression of oncogenic fusion proteins that retain the kinase domain of the proto-oncogene, and the downstream signaling directs cells to proliferation and survival in a ligand-inde-

Correspondence: Luis Mendoza, Pernerova 691/42 Praha 8, 18600 Prague, Czech Republic.

E-mail: luis.mendoza@iqvia.com

Key words: Non-small cell lung cancer; RET-rearrangement; clinical trials; review; tyrosine kinase.

Conflict of interest: the author declares no potential conflict of interest.

Received for publication: 12 January 2018.

Accepted for publication: 20 June 2018.

This work is licensed under a Creative Commons Attribution NonCommercial 4.0 License (CC BY-NC 4.0).

(C) Copyright L. Mendoza., 2018

Licensee PAGEPress, Italy

Oncology Reviews 2018; 12:352

doi:10.4081/oncol.2018.352 pendent manner. Inhibition of the oncogenic fusion proteins can result in potent cancer growth inhibition and regression of tumors in patients. To date, the drugs for NSCLC approved by the U.S. Food and Drug Administration (FDA) and the European Medicines Agency (EMA) have been targeted to ALK and ROS1 rearrangements only. The activity of many multi-kinase inhibitors has been explored in RET-rearranged NSCLC, and novel RETspecific inhibitors have recently transitioned to clinical development. Based on initial results obtained with the multi-kinase inhibitors in RET-rearranged NSCLC, the National Comprehensive Cancer Center Network (NCCN) 2017 guidelines recommend the use of cabozantinib and vadentanib outside the context of a clinical trial. In this paper, we review the current available clinical data on RET inhibitors, reasons for their resistance, and emerging treatment approaches in RET-rearranged NSCLC.

\section{$R E T$ rearrangements in NSCLC}

RET is a $150 \mathrm{KDa}$ membrane-bound receptor tyrosine kinase that is expressed in a variety of neuronal and endocrine tumors. ${ }^{5}$ The RET transmembrane protein is encoded by proto-oncogene RET located on chromosome 10q11.2. ${ }^{6}$ Activation of RET leads to auto-phosphorylation on intracellular tyrosine residues and initiation of Ras/MAP kinase, PI3K/AKT, and phospholipase C pathways that signal cell proliferation and survival. Oncogene activation of RET can occur by somatic or germline alterations. Germline mutations of RET lead to type 2 multiple endocrine neoplasia, whereas somatic mutations lead to sporadic medullary thyroid carcinoma. Somatic RET rearrangements induce formation of the RET fusion protein kinases that localize in the cytosol and have transforming and oncogenic properties. ${ }^{7}$ Fusion proteins resulting from the chromosomal rearrangement of RET were first identified in papillary thyroid carcinoma (PTC). ${ }^{8,9}$ In 2012, four independent research groups identified RET fusions in NSCLC. ${ }^{10-}$ ${ }^{13}$ Collectively, these studies concluded that RET fusions occur in approximately $1 \%$ to $2 \%$ of NSCLCs and that RET rearrangements tend to be mutually exclusive with other major lung-cancer drivers such as EGFR, KRAS mutations and $A L K$ or ROS1 rearrangements. ${ }^{14}$ In NSCLC, at least 12 fusion $R E T$ partner genes have been identified to date. The recent global registry of patients with RET-rearranged NSCLC reported that among 81 cases with identifiable fusion partners, $72 \%$ involved the kinesin family $5 B$ gene $(K I F 5 B)$. The second most common fusion partner is CCDC6 (23\%), followed by NCOA4 (2\%), EPHA5 (1\%) and PICALM (1\%). ${ }^{15}$ RET rearrangements were observed in males and females in equal proportions. As per the global registry, $63 \%$ were never smokers, 24\% were former smokers, and $10 \%$ were current smokers. Histologically, most RET rearrangements were identified in adenocarcinoma. At present, there is no gold-standard method for the identification of RET rearrangements. Although immuno- 
histochemistry (IHC) is an effective screening tool to detect ALKand ROS1-positive NSCLC, the utility of IHC for the detection of $R E T$ fusions has been limited because of variable staining patterns and weak reactivity. ${ }^{16}$ Reverse transcriptase polymerase chain reaction (RT-PCR) is both sensitive and specific for the detection of known fusions, but it is not reliable for the detection of new fusion partners. Fluorescence in situ hybridization (FISH) and next-generation sequencing (NGS) are effective techniques for the detection of RET fusions, but their high costs and technical expertise for interpretation made them usually available only in larger reference centers. ${ }^{17}$ Therefore, in most screening studies for RET rearrangements, RT-PCR was typically combined with FISH, suggesting that they are complementary.

\section{Clinical trial results with RET inhibitors for $R E T$ rearrangements in NSCLC}

The main clinical data on the most developed multi-kinase inhibitors in RET-rearranged NSCLC are summarized in Tables 1 and 2. The clinical activity of RET-directed therapy was first reported in 2013 by Drillon et al., when three patients with RETrearranged NSCLC were treated with cabozantinib. ${ }^{18}$ Two of these patients experienced partial responses by RECIST 1.1 criteria, and the third had prolonged stable disease. Based on this early experience, a phase 2 trial was conducted to assess the activity of cabozantinib $60 \mathrm{mg} / \mathrm{d}$ in 26 patients with $R E T$-rearranged NSCLC screened by FISH or NGS. Of these patients, $62 \%$ had a KIF5BRET rearrangement. Among 25 patients who were assessable for response, there were seven partial responses [overall response rate (ORR) 28\%]. The median progression-free survival (mPFS) was 5.5 months, and the median overall survival (mOS) was 9.9 months. ${ }^{19}$ The ORR in patients with KIF5B-RET-rearranged NSCLC was $20 \%$, and it was $50 \%$ in patients with different known RET fusion genes. Twenty-six patients treated were evaluable for toxicity. Treatment-related adverse events were predominantly grade 1 or grade 2 , and one or more drug-related toxicities of any grade were observed in 25 patients (overall toxicity rate of $96.2 \%$ ). The most common treatment-related adverse events of any grade were increased alanine aminotransferase (ALT), increased aspartate aminotransferase (AST), hypothyroidism, diarrhea, palmar plantar erythrodysesthesia, and skin hypopigmentation. The most common grade 3 treatment-related adverse events were lipase elevation in four patients $(15 \%)$, increased ALT in two patients $(8 \%)$, decreased platelet count in two patients $(8 \%)$, and hypophosphatemia in two patients $(8 \%)$. Patients in whom these toxicities were observed were asymptomatic. Nineteen patients $(73 \%)$ required a cabozantinib dose reduction due to intolerable grade 2 or grade 3 drug-related toxicities. The most common reasons for dose reduction included palmar plantar erythrodysesthesia in seven patients $(37 \%)$, fatigue in three patients $(16 \%)$, and diarrhea in two patients $(11 \%)$

In selected patients with NSCLC, vandetanib (300 mg/d) was tested in two different trials. A Japanese phase II (LURET) study included 1,536 patients with EGFR-negative NSCLC, who were screened by multiplex transcriptase PCR and FISH break-apart assay. ${ }^{20}$ Among the patients who were screened, 34 (2\%) were RET positive, and 19 were enrolled in the study and treated with $300 \mathrm{mg}$ of vandetanib daily. Among 17 patients with evaluable data included in primary analysis, the ORR was $53 \%$, and the median PFS was 4.7 months. The OS rate at 12 months was $47 \%$, and the median OS was 11.1 months. The treatment response and survival outcome were much higher in patients with the CCDC6-RET fusion subtype, with $83 \%$ ORR and mPFS of 8.3 months compared with $20 \%$ and 2.9 months, respectively, for patients with the KIF5BRET fusion variant. In another similar study design, a Korean phase II trial evaluated vandetanib $(300 \mathrm{mg} / \mathrm{d})$ in 18 patients with RET-rearranged NSCLC; $28 \%$ of them had KIF5B-RET rearrangement, $11 \%$ were $C C D C 6-R E T$-positive, $56 \%$ had an unknown $R E T$ fusion gene, and one patient (5\%) displayed a novel MYO5C-RET rearrangement. Among the 17 patients with evaluable results, the ORR was $18 \%$ (three patients with partial responses), the mPFS was 4.5 months, the mOS was 11.6 months and the 1 -year OS rate was $33 \% .^{21}$ Overall, the treatment was well tolerated. Hypertension (16.89\%), rash (13.72\%), diarrhea (8.44\%), acne

Table 1. Clinical data on single-agent RET inhibitors in advanced pre-treated RET-rearranged NSCLC.

\begin{tabular}{|c|c|c|c|c|c|c|}
\hline Type of Study (Identifier) & RET inhibitor & Screening techniques & \# patients & ORR & $\begin{array}{c}\text { mPFS } \\
\text { (months) }\end{array}$ & $\begin{array}{c}\text { mOS } \\
\text { (months) }\end{array}$ \\
\hline Phase II single arm (NCT01639508) ${ }^{19}$ & Cabozantinib $60 \mathrm{mg} / \mathrm{d}$ & FISH or NGS & 26 & $28 \%$ & 5.5 & 9.9 \\
\hline Phase II single arm - Japan (UMIN000010095) ${ }^{20}$ & Vandetanib $300 \mathrm{mg} / \mathrm{d}$ & RT-PCR and FISH & 19 & $53 \%$ & 4.7 & 11.1 \\
\hline Phase II single arm (NCT01823068)21 & Vandetanib $300 \mathrm{mg} / \mathrm{d}$ & FISH & 18 & $18 \%$ & 4.5 & 11.6 \\
\hline Phase II single arm (NCT01877083)22 & Lenvatinib $24 \mathrm{mg} / \mathrm{d}$ & NA & 25 & $16 \%$ & 7.3 & NR \\
\hline
\end{tabular}

NSCLC, non-small cell lung cancer; RET, rearranged during transfection; RET, rearranged during transfection gene; FISH, fluorescence in situ hybridization; NGS, next-generation sequencing; RT-PCR, reverse-transcriptase polymerase chain reaction; ORR, objective response rate; $\mathrm{mPFS}$, median progression-free survival; mOS, median overall survival; NR, not reached; NA, not available.

Table 2. The most common treatment-emergent adverse events (TEAEs) of the most developed RET inhibitors in NSCLC.

\begin{tabular}{|c|c|c|}
\hline Cabozatinib $(n=26)$ & Vandetanib $(n=18)$ & Lenvatinib $(n=25)$ \\
\hline ALT increased (96\%) & Hypertension (89\%) & Hypertension (68\%) \\
\hline AST increased (73\%) & Rash (72\%) & Nausea $(60 \%)$ \\
\hline Hypothyroidism (69\%) & Diarrhea (44\%) & Decreased appetite (52\%) \\
\hline Diarrhea (62\%) & Acne (28\%) & Diarrhea (52\%) \\
\hline Palmar plantar erythrodysesthesia (58\%) & Xerosis (22\%) & Proteinuria (48\%) \\
\hline Skin hypopigmentation (50\%) & Abdominal discomfort (17\%) & Vomiting (44\%) \\
\hline Dose reduction $(73 \%)$ & Dose reduction (28\%) & Dose reduction (64\%) \\
\hline
\end{tabular}

NSCLC, non-small cell lung cancer; RET, rearranged during transfection; ALT, alanine aminotransferase; AST, aspartate aminotransferase; n, number of subjects. 
(5.28\%), xerosis $(4.22 \%)$, and abdominal discomfort $(3.17 \%)$ were the most frequent adverse events in the study patients (Table 2). Five patients experienced adverse events of grade 3: hypertension (3, 18\%), asymptomatic QTc prolongation in electrocardiography $(2,12 \%)$, and elevated serum level of aminotransferases $(1,6 \%)$. Among these, four patients underwent dose reduction (28\%).

In another phase II trial, lenvatinib $(24 \mathrm{mg} / \mathrm{d})$ was tested in 25 patients with RET-rearranged NSCLC. The results were presented at the 2016 European Society for Medical Oncology (ESMO) Congress. Of them, $52 \%$ had a KIF5B-RET rearrangement and $48 \%$ had different unknown RET fusions genes determined by NGS. The ORR was $16 \%$ (four patients with partial responses) and the mPFS was 7.3 months. ${ }^{22}$ In seven patients who had received previous RET therapy, ORR with lenvatinib was $14 \%$ with a mPFS lower than other knowns fusion variants (3.6 versus 9.1 months). Grade $\geq 3$ treatment emergent adverse events (TEAEs) occurred in $23(92 \%)$ patients. Of three fatal AEs, one (pneumonia) was possibly related to lenvatinib. TEAEs requiring dose reduction occurred in $16(64 \%)$ patients. The most common TEAEs included hypertension $(17.68 \%)$, nausea $(15.60 \%)$, decreased appetite $(13.52 \%)$, diarrhea $(13.52 \%)$, proteinuria (48\%), and vomiting (11.44\%).

Other multi-target kinase inhibitors have also been tested in RET-rearrangement NSCLC, including sunitinib, ${ }^{23}$ sorafenib, ${ }^{24}$ alectinib, ${ }^{25}$ nintedanib, ponatinib and regorafenib. ${ }^{26}$ Data on these agents are generally limited to case reports. No direct comparison of RET inhibitors has been performed. Therefore, it is not possible to identify the most active RET inhibitor based on the currently available clinical data.

\section{Mechanisms of RET-inhibitor resistance and}

\section{emerging therapeutic approach}

The activity of multi-kinase inhibitors in patients with RETrearranged NSCLC (ORR 16\%-53\% and mPFS 4.5-7.3) is clearly inferior to the responses and survival outcomes seen with selective TKIs in other oncogene-associated NSLC models. In fact, the ORR of $56 \%-85 \%$ and mPFS duration 9.2-13.7 months was achieved with targeted TKIs in patients with EGFR mutant, ${ }^{27}$ the ORR of $60 \%-95 \%$ and mPFS 8-11 months was achieved in patients with $A L K$-rearranged NSCLC, 28 and the ORR of $65 \%$ $85 \%$ and mPFS 9.3-19.3 months was achieved in patients with ROS1-rearragend NSCLC. ${ }^{29}$ One possible explanation for the limited efficacy of multi-kinase RET inhibitors relates to the inhibition of non-RET kinases rather than RET-specific blocking. As such, the use of multi-kinase RET inhibitors is often associated with high rate of toxicities (hypertension, proteinuria, palmar plantar erythodysesthesia) that are mostly due to the activity against VEGFR kinases, or diarrhea due to activities related to EGFR inhibition, which lead to dose reductions in up to $73 \%$ of the patients (Table 2) and achieving suboptimal RET-inhibitory plasma concentrations consequently. It is therefore possible that other intrinsic mechanisms play a role in the resistance.

The mechanisms of acquired resistance to RET inhibitors in the patients are currently poorly understood. In fact, the Japanese phase II study of vandetanib showed a lower ORR and shorter mPFS duration among patients with tumors harboring the KIF5BRET-positive fusion versus those with tumors harboring the CCDC6-RET fusion type. ${ }^{20}$ Recent molecular studies have identified MDM2 proto-oncogene (MDM2) amplification in pretreatment biopsy specimens from 8 of 16 NSCLC who developed resistance to cabozantinib. ${ }^{30}$ Metastasis to the central nervous system (CNS) also represents an important clinical challenge in RETrearranged NSCLC. Vandetanib is thought to have a limited blood-

Table 3. Ongoing clinical trials of known and novel RET inhibitors in RET-rearranged NSCLC.

\begin{tabular}{|c|c|c|c|c|c|}
\hline Agent & Manufacturer & $\begin{array}{l}\text { Anti-RET } \\
\left(\mathrm{IC}_{50}, \mathrm{nM}\right)\end{array}$ & Other major targets & Phase & NSCLC population (Identifier) \\
\hline Alectinib & Roche & 4.8 & ALK, LTK, CHEK2, FLT3, PHKG2 & $\mathrm{I} / \mathrm{II}$ & RET-rearranged NSCLC - Japan (UMIN000020628) \\
\hline Alectinib & Roche & 4.8 & ALK, LTK, CHEK2, FLT3, PHKG2 & $\mathrm{I} / \mathrm{II}$ & RET-rearranged NSCLC (NCT03131206) \\
\hline Apatinib & $\begin{array}{l}\text { Jiangsu Hengrui/ } \\
\text { LSK BioPharma }\end{array}$ & 13 & VEGFR2, KIT, SRC & II & RET-rearranged NSCLC (NCT02540824) \\
\hline BLU-667 & Blueprint Medicines & 0.4 & VEGFR2 & $\mathrm{I} / \mathrm{II}$ & $\begin{array}{l}\text { RET-rearranged NSCLC prior or not prior to TKI that } \\
\text { inhibits RET (NCT03037385) }\end{array}$ \\
\hline Lenvatinib & Eisai & 1.5 & VEGFR1-3, FGFR1-4, PDGFR, KIT & II & $\begin{array}{l}\text { KIF5B-RET-positive and other confirmed RET } \\
\text { translocations lung adenocarcinoma } \\
\text { (NCT01877083) }\end{array}$ \\
\hline Ponatinib & Ariad & 25.8 & BCR-ABL, SRC, VEGFR, PDGFR, FGFR, FLT3, KIT & II & RET-rearranged NSCLC (NCT01935336) \\
\hline Sunitinib & Pfizer & $220-1300$ & VEGFR1-2, PDGFR $\beta$, FLT3, KIT & II & RET-positive lung adenocarcinoma (NCT01829217) \\
\hline Sitravatinib & Mirati Therapeutics & 44 & $\begin{array}{l}\text { VEGFR, PDGFR } \alpha, \text { MET, AXL, TRK, DDR1-2, FLT3, } \\
\text { KIT, EPHA2-4, EPHB2/4, MER, MST1R }\end{array}$ & $\mathrm{I} / \mathrm{IB}$ & $\begin{array}{l}\text { NSCLC with genetic alterations in MET, AXL, RET, } \\
\text { TRK, DDR2, KDR, PDGFRa, KIT or CBL } \\
\text { (NCT02219711) }\end{array}$ \\
\hline BLU-667 & Blueprint Medicines & 0.4 & RET-specific inhibitor & $\mathrm{I} / \mathrm{II}$ & $\begin{array}{l}\text { RET-rearranged NSCLC prior or not prior to TKI that } \\
\text { inhibits RET (NCT03037385) }\end{array}$ \\
\hline LOXO-292 & $\begin{array}{l}\text { Loxo } \\
\text { Oncology }\end{array}$ & $\mathrm{NA}$ & RET-specific inhibitor & I & RET-fusion NSCLC (NCT03157128) \\
\hline \multicolumn{6}{|c|}{$\begin{array}{l}\text { NSCLC, non-small cell lung cancer; RET, rearranged during transfection; RET, rearranged during transfection gene, IC50, half maximal inhibitory concentration; VEGFR, vascular endothelial growth factor receptor; MET, } \\
\text { MET proto-oncogene, receptor tyrosine kinase; AXL, AXL receptor tyrosine kinase; FLT3, FMS-like tyrosine kinase; KIT, KIT proto-oncogene, receptor tyrosine kinase; FGFR, fibroblast growth factor receptor; PDGFR, } \\
\text { platelet-derived growth factor receptor; BCR-ABL, breakpoint cluster region-Abelson murine leukemia viral oncogene homolog 1; SRC, SRC proto-oncogene, non-receptor tyrosine kinase; DDR, discoidin domain recep- } \\
\text { tor; EPHA, ephrin receptor A; EPHB, ephrin receptor B; MSTIR, macrophage-stimulating protein receptor 1; PDGRF, platelet-derived growth factor receptor; PDGRFa, platelet-derived growth factor receptor alpha gene; } \\
\text { PDGFRb, platelet-derived growth factor receptor beta gene; KIT, KIT proto-oncogene receptor tyrosine kinase gene; ALK, anaplastic lymphoma kinase; LTK, leukocyte receptor tyrosine kinase; CHEK2, checkpoint kinase } \\
\text { 2; PHKG2, phosporylase kinase gamma 2; MER, MER tyrosine kinase receptor; MSTIR, macrophage stimulating } 1 \text { receptor; TRK, tropomyosin receptor kinase; KDR, kinase insert domain receptor; CBL, Casitas B-lineage } \\
\text { Lymphoma. }\end{array}$} \\
\hline
\end{tabular}


brain barrier penetration, which may be improved by modulation of P-gp/Abcb1- and Bcrpl/Abcg2-mediated efflux through the use of mTOR inhibitor. ${ }^{31,32}$ Therefore, a phase I trial (NCT01582191) testing the antitumor activity of vandetanib $(100 \mathrm{mg} / \mathrm{d})$ in combination with everolimus (m-TOR inhibitor) at $2.5 \mathrm{mg} / \mathrm{d}$ in patients who have refractory solid tumors, including those with RETrearranged NSCLC, was initiated. Preliminary results indicate that the combination of vandetanib and everolimus is well tolerated. The most common treatment-related AEs are diarrhea, fatigue, mucositis, and rash. The combination produced significant antitumor activity in patients with RET-rearranged NSCLC. ${ }^{33}$ This study is active and recruiting patients; updated results about a larger cohort of patients with RET-rearranged NSCLC are eagerly awaited.

Currently, there are several ongoing clinical trials testing the safety and efficacy of known and novel RET inhibitors in RETrearranged NSCLC (Table 3). The difference in the relative potency of RET inhibitors restricts the therapeutic window as high levels of these drugs may cause toxicity related to other targets before reaching optimum RET inhibition levels. Therefore, novel and potent inhibitors have been developed to selectively target the RET kinase. Two of these novel RET-specific inhibitors, BLU-667 and LOXO-292, ${ }^{34,35}$ have broad preclinical activity against various RET rearrangements. BLU-667 is being studied in a phase I trial (NCT03037385) enrolling medullar thyroid carcinoma (MTC), NSCLC and other tumors displaying RET activation. The trial has a target enrollment of 115 patients. Preliminary data from the trial is expected in Q1 2018. LOXO-292 is currently being studied in a phase I trial (NCT03157128) enrolling advanced tumors with RET drivers, including NSCLC with RET fusions. The trial is seeking to enroll 180 patients, and an early update from the trial was presented at the International Association for the Study of Lung Cancer (IASLC) $18^{\text {th }}$ World Conference on Lung Cancer 2017 in Yokohama, Japan. The abstract describes the first two patients with RET-fusion lung cancer with and without brain metastases treated with LOXO-292. Both patients had disease progression while receiving prior multi-kinase inhibitors. On LOXO-292, both patients achieved partial responses and the therapy has been welltolerated with no adverse events attributed to it. A more robust data of the trial is expected in 2018 .

\section{Conclusions}

At present, the use of the few investigated RET inhibitors in RET-rearranged NSCLC has not shown striking clinical benefits compared with what has been seen with other targeted agents in other gene rearrangement types. The mechanisms of resistance to them are not well understood. Furthermore, RET inhibitors showed high rates of severe toxicities, leading to frequent dose reduction. No definitive conclusions can be done on the rather confounding data about the potential different impact of anti-RET therapies according the RET-rearrangement or fusion variants generated from small subgroups analysis. RET-rearrangement remains a challenging target, and the biology behind these drivers in NSCLC will require further exploration of the most potent and selective RET inhibitors and CNS activity. The RET rearrangements are rather rare, so that it may not be possible to conduct randomized trials to compare the activity of traditional chemotherapy or immunotherapy or the impact on different fusion variants. To conduct prospective trials with larger sample sizes, collaboration between various investigators and centers around the globe is crucial. Combination therapies and novel RET-specific inhibitors in NSCLC patients harboring RET rearrangements are being explored to boost the activity observed with the existing multikinase RET inhibitors in the clinic. Further research in the field of RET-inhibitors in RET-rearranged NSCLC is encouraged.

\section{References}

1. Scagliotti G, Stahel RA, Rosell R, et al. Alk translocation and crizotinib in non-small cell lung cancer: an evolving paradigm in oncology drug development. Eur J Cancer 2012;48:961-73.

2. Bergethon K, Shaw AT, Ou SH, et al. ROS1 rearrangements define a unique molecular class of lung cancers. J Clin Oncol 2012;30:863-70.

3. Vaishnavi A, Capelletti M, Le AT, et al. Oncogenic and drugsensitive NTRK1 rearrangements in lung cancer. Nat Med 2015;19:1469-72.

4. Kohno T, Nakaoku T, Tsuta K, et al. Beyond ALK-RET, ROS1 and other oncogene fusions in lung cancer. Transl Lung Cancer Res 2015;4:156-64.

5. Arighi E, Borrello MG, Sariola H. RET tyrosine kinase signaling in development and cancer. Cytokine Growth Factor Rev 2005;16:441-67.

6. Ishizaka Y, Itoh F, Tahira T, et al. Human ret proto-oncogene mapped to chromosome 10q11.2. Oncogene 1989;4:1519-21.

7. Mulligan LM. RET revisited: expanding the oncogenic portfolio. Nat Rev Cancer 2014;14:173-86.

8. Bongarzone I, Pierotti MA, Monzini N, et al. High frequency of activation of tyrosine kinase oncogenes in human papillary thyroid carcinoma. Oncogene 1989;4:1457-62.

9. Grieco M, Santoro M, Berlingieri MT, et al. PTC is a novel rearranged form of the ret proto-oncogene and is frequently detected in vivo in human thyroid papillary carcinomas. Cell 1990;60:557-63.

10. Takeuchi K, Soda M, Togashi Y, et al. RET, ROS1 and ALK fusions in lung cancer. Nat Med 2012;18:378-81.

11. Ju YS, Lee WC, Shin JY, et al. A transforming KIF5B and RET gene fusion in lung adenocarcinoma revealed from wholegenome and transcriptome sequencing. Genome Res 2012;22:436-45.

12. Kohno T, Ichikawa H, Totoki Y, et al. KIF5B-RET fusions in lung adenocarcinoma. Nat Med 2012;18:375-7.

13. Lipson D, Capelletti M, Yelensky R, et al. Identification of new ALK and RET gene fusions from colorectal and lung cancer biopsies. Nat Med 2012;18:382-4.

14. Kato S, Subbiah V, Marchlik E, et al. RET aberrations in diverse cancers: next generation sequencing of 4.871 patients. Clin Cancer Res 2017;23:1988-97.

15. Gautschi O, Milia J, Filleron T, et al. Targeting RET in Patients With RET-Rearranged Lung Cancers: Results From the Global, Multicenter RET Registry. J Clin Oncol 2017;35:140310.

16. Gautschi O, Milia J, Filleron T, et al. Diagnostic method for the detection of KIF5B-RET transformation in lung adenocarcinoma. Lung Cancer 2013;82:44-50.

17. Tsuta K, Kohno T, Yoshida A, et al. RET-rearranged nonsmall-cell lung carcinoma: a clinicopathological and molecular analysis. Br J Cancer 2014;110:1571-8.

18. Drilon A, Wang L, Hasanovic A, et al. Response to Cabozatinib in patients with RET fusion-positive lung adenocarcinomas. Cancer Discov 2013;3:630-5.

19. Drilon A, Rekhtman N, Arcila M, et al. Cabozatinib in patients with advanced RET-rearranged non-small-cell-lung-cancer: an open-label, single centre, phase 2, single-arm trial. Lancet Oncol 2016;17:1653-60. 
20. Yoh K, Seto T, Satouchi M, et al. Vandetanib in patients with previously treated RET-rearranged advanced non-small-cell lung cancer (LURET): an open-label, multicentre phase 2 trial. Lancet Respir Med 2017;5:42-50.

21. Lee SH, Lee JK, Ahn MJ, et al. Vandetanib in pretreated patients with advanced non-small cell lung cancer-harboring RET rearrangement: a phase II clinical trial. Ann Oncol 2017;28:292-7.

22. Velcheti V, HidaT, Reckamp KL, et al. Phase 2 study of lenvatinib (LN) in patients (Pts) with RET fusion-positive adenocarcinoma of the lung. Ann Oncol 2016;27:416-54.

23. Wu H, Shih JY, Yang JC. Rapid response to sunitinib in a patient with lung adenocarcinoma harboring KIF\%B-RET fusion gene. J Thorac Oncol 2015;10:e95-6.

24. Horiike A, Takeuchi K, Uenami T, et al. Sorafenib treatment for patients with RET fusion-positive non-small-cell-lung-cancer. Lung Cancer 2016;9:43-6.

25. Lin JJ, Kennedy E, Sequist LV, et al. Clinical activity of alectanib in advanced RET-rearranged non-small-cell-lungcancer. J Thorac Oncol 2016;11:2027-32.

26. Gautschi O, Milia J, Filleron T, et al. Targeting RET in patients with RET-rearranged lung cancers: Results from the global, mukticenter RET registry. J Clin Oncol 2017;35:1403-10.

27. Castellanos E, Feld E, Horn L Driven by mutations: the predictive value of mutations subtype in EGFR-mutated non-small cell lung cancer. J Thorac Oncol 2017;12:612-23.

28. Caccese M, Ferrara R, Pilotto S, et al. Current and developing therapies for the treatment of non-small cell lung cancer with ALK abnormalities; update and perspectives for clinical practice. Exp Opin Pharmacother 2016;17:2253-66.
29. Facchinetti F, Rossi G, Bria E, et al. Oncogene addiction in non-small cell lung cancer: focus on ROS1 inhibition. Cancer treat Rev 2017;55:83-95.

30. Somwar R, Smith R, Hayashi T, et al MDM2 amplification (Amp) to mediate cabozantinib resistance in patients (Pts) with advanced RET-rearranged lung cancers. J Clin Oncol 2016;34:15: abstr 9068.

31. Subbiah V, Berry J, Roxas M, et al. Systemic and CNS activity of the RET inhibitor vandetanib combined with the mTOR inhibitor everolimus in KIF5B-RET re-arranged non-small cell lung cancer with brain metastasis. Lung Cancer 2015;89:76-9.

32. Minocha M, Khurana V, Qin B, et al. Co-administration strategy to enhance brain accumulation of vandetanib by modulating P-glycoprotein (P-gp/Abcb1) and breast cancer resistance protein (Bcrp1/Abcg2) mediated efflux with m-TOR inhibitors. Int J Pham 2012;434:306-14.

33. Cascone T, Hess KR, Piha-Paul SA, et al. Safety, toxicity and activity of multi-kinase inhibitor vandetanib in combination with everolimus in advanced solid tumors. J Clin Oncol 2016;34: abstr 9073.

34. Rahal R, Evans EK, Hu W, et al. The development of potent, selective RET inhibitors that target both wild type RET and prospectively identified resistance mutations to multi-kinase inhibitors (abstract). Cancer Res 2016;76: abstr 2641.

35. Brandhuber BJ, Nanda N, Haas J, et al. Identification and characterization of highly potent and selective RET kinase inhibitors for the treatment of RET-driven cancer. Cancer Res 2015;14: abstr B192. 